\title{
Hippocampal damage and memory impairments as possible early brain complications of type 2 diabetes
}

\author{
S. M. Gold • I. Dziobek • V. Sweat • A. Tirsi • \\ K. Rogers $\cdot$ H. Bruehl $\cdot$ W. Tsui $\cdot$ S. Richardson • \\ E. Javier • A. Convit
}

Received: 23 October 2006 / Accepted: 9 January 2007 / Published online: 14 February 2007

(C) Springer-Verlag 2007

\begin{abstract}
Aims/hypothesis There is evidence that type 2 diabetes mellitus is associated with cognitive impairment. Most studies investigating this association have evaluated elderly individuals, after many years of diabetes, who generally have poor glycaemic control and significant vascular disease. The aim of the current study was to investigate the early cognitive consequences and associated brain correlates of type 2 diabetes.

Materials and methods With regard to cognition and brain measures, we compared 23 age-, sex- and educationmatched control subjects with 23 mostly middle-aged individuals with relatively well-controlled diabetes of less than 10 years from the time of diagnosis.

Results We found deficits in hippocampal-based memory performance and preservation of other cognitive domains. Relative to control subjects, individuals with diabetes had reductions in brain volumes that were restricted to the hippocampus. There was an inverse relationship between glycaemic control and hippocampal volume; in multivariate
\end{abstract}

S. M. Gold and I. Dziobek contributed equally to this work

S. M. Gold · I. Dziobek · V. Sweat · A. Tirsi · K. Rogers •

H. Bruehl $\cdot$ W. Tsui $\cdot$ E. Javier $\cdot$ A. Convit $(\bowtie)$

Department of Psychiatry,

New York University School of Medicine,

550 First Avenue, HN-400,

New York, NY 10016, USA

e-mail: antonio.convit@med.nyu.edu

S. Richardson

Department of Medicine,

New York University School of Medicine,

New York, NY 10016, USA

W. Tsui $\cdot$ A. Convit

Nathan Kline Institute for Psychiatric Research,

Orangeburg, NY 10962, USA regression analysis, $\mathrm{HbA}_{1 \mathrm{c}}$ was the only significant predictor of hippocampal volume, accounting for $33 \%$ of the observed variance. Other variables commonly associated with type 2 diabetes, such as elevated BMI, hypertension or dyslipidaemia, did not independently contribute to the variance in hippocampal volume.

Conclusions/interpretation These results suggest that the medial temporal lobe may be the first brain site affected by type 2 diabetes and that individuals in poorer metabolic control may be affected to a greater extent.

Keywords Cognition · Diabetes · Hippocampus ·

Mechanism $\cdot$ Memory $\cdot$ Type 2 diabetes

$\begin{array}{ll}\text { Abbreviations } \\ \text { CSF } & \text { cerebrospinal fluid } \\ \text { FOV } & \text { field of view } \\ \text { ICV } & \text { intracranial vault volume } \\ \text { MIDAS } & \begin{array}{l}\text { Multimodal Image Data Analysis System } \\ \text { MRI }\end{array} \\ \text { magnetic resonance imaging } \\ \text { NCEP } & \text { National Cholesterol Education Program } \\ \text { ROI } & \text { regions of interest } \\ \text { VBM } & \text { voxel-based morphometry } \\ \text { WMH } & \text { white matter hyperintensity } \\ \text { WMS-R } & \text { Wechsler Memory Scale-Revised }\end{array}$

Introduction

The number of Americans with type 2 diabetes has been rising in parallel with the increasing prevalence of obesity [1]. There is accumulating evidence that diabetes, in addition to increasing the risk of cardiovascular disease, stroke and kidney failure, may also adversely affect brain function independently from atherosclerotic disease. To 
date, numerous studies have demonstrated that type 2 diabetes is associated with cognitive deficits (for example [2-5]).

In line with the growing literature demonstrating cognitive impairments, evidence suggests that type 2 diabetes is associated with damage to the brain. A few studies employing in vivo brain imaging have reported 'accelerated brain ageing' in patients with diabetes (for example [4, 6]). In addition, among individuals with type 2 diabetes, reductions in cognitive functioning have been reported to be associated with white matter lesions, atrophy and the presence of infarcts [4]. However, it should be noted that these researchers used qualitative ratings. Investigators studying cases from a large epidemiological sample have shown that, relative to nondiabetic control subjects, elderly (average age 77.8 years) individuals with type 2 diabetes exhibit reductions in hippocampal and amygdalar volumes [7]; however, no neuropsychological data were reported in this sample. A moderately elevated risk of hippocampal atrophy (odds ratio $1.7,95 \%$ CI $0.9-2.9$ ) has been demonstrated among very old men with type 2 diabetes [8], but it should be noted that this study included individuals with dementia, Alzheimer's disease, strokes, lacunes and high rates of white matter disease.

Cognitive problems associated with the disease have traditionally been assumed to be due to atherosclerosis [9, 10], since type 2 diabetes is often linked to peripheral vascular disease (for example [11]) and dyslipidaemia (for example [12-14]). These diabetes-associated factors appear to influence the brain even in healthy individuals. For example, obesity has been related to cognitive problems [15] and age-associated brain atrophy [16]. Similarly, hypertension has been associated with global or frontal atrophy [17] and cognitive dysfunction [18].

Diabetes itself may directly affect the brain. For example, improvements in diabetes control have been associated with improvements in cognitive functioning $[19,20]$. In addition, deficits in learning and memory have also been described among non-diabetic individuals with insulin resistance (for example [21, 22]), even after accounting for the possible effects of atherosclerosis [23].

The aim of the present study was to examine individuals who, as a group, had been diagnosed with diabetes fewer than 10 years previously, and had fairly good glycaemic control. Subjects in this sample were free of obvious vascular disease and were, on average, 60 years of age, and had therefore not yet entered the years of increased risk for cognitive impairment from sporadic Alzheimer's disease. We investigated the impact of type 2 diabetes and overall glycaemic control $\left(\mathrm{HbA}_{1 \mathrm{c}}\right.$ levels) on the brain, and assessed whether obesity, dyslipidaemia and hypertension modify these associations.

\section{Subjects and methods}

Subjects

The participants represent a sample of convenience, consisting of consecutive cases evaluated as part of a federally sponsored study. Individuals with diabetes were referred by endocrinologists or responded to advertisements on the internet or in local periodicals. Control subjects were selected from our ongoing normal ageing studies. Participants were between 45 and 70 years of age, and had completed a minimum of 12 years of education. Study subjects underwent medical, endocrine, neurological, psychiatric, neuropsychological and brain magnetic resonance imaging evaluations. All participants gave informed written consent, and the study was approved by the local institutional board of research associates.

Participants with type 2 diabetes mellitus We evaluated 23 consecutive individuals with one of the following: a fasting blood glucose level $>6.94 \mathrm{mmol} / \mathrm{l}$; a $2 \mathrm{~h}$ glucose level $>11.1 \mathrm{mmol} / 1$ during an OGTT; or a prior diagnosis of type 2 diabetes. To avoid the possible confounding effects of hypoglycaemic episodes, we excluded individuals treated with insulin or insulin secretagogues. In addition, given that microvascular insufficiency can be present in type 2 diabetes, we assessed postural colour changes in the lower extremities as part of our clinical screening. None of our study participants had clinical findings consistent with gross microvascular insufficiency. However, patients were not tested for the presence of subclinical diabetic retinopathy.

Control subjects Twenty-three healthy control subjects were selected who matched the participants with type 2 diabetes in terms of age, education and sex. Selection of subjects was made blind with regard to their cognitive and MRI evaluations. Individuals were selected as control subjects if their fasting glucose and fasting insulin values were $<5.55 \mathrm{mmol} / 1$ and $<104.18 \mathrm{pmol} / \mathrm{l}$, respectively. It was not possible to identify control subjects who matched our diabetic subjects with regard to BMI and who did not have elevated fasting insulin levels, thus providing evidence of insulin resistance.

Neuropsychological and psychiatric assessment

Recent memory was assessed with the California Verbal Learning Test (short and long delay scores) [24]. The whole Wechsler Memory Scale-Revised (WMS-R) [25] was administered, from which the general and delayed indices were used. Because of their learning component, we also 
administered the Immediate and Delayed Paragraph Recall subtests from the Guild Memory Test [26].

Working memory was tested using the Digit Span Backward subtest from the WMS-R. Attention was assessed with the Perceptual Speed Test (a cancellation task) and the Digit Symbol Substitution Test (DSST). The ability to monitor and inhibit responses (one aspect of executive function) was measured using the interference score of the Stroop task [27]. A measure of general intellectual functioning was assessed using the Shipley Institute of Living Scale [28]; scores on this scale were used to estimate IQ scores [29].

\section{Exclusion criteria}

All participants completed the National Institute of Mental Health Quick Diagnostic Interview Schedule screening version [30]. Evidence of neurological, medical (other than diabetes, dyslipidaemia or hypertension), or psychiatric (including alcohol or other substance abuse or depression) signs and symptoms that allowed for a diagnosis to be made excluded individuals from participation in the study. Depression was further evaluated using the Hamilton Depression Scale [31].

All subjects were screened using the Rosen modification of the Hachinski ischaemia scale [32]. The scale includes items such as history of hypertension, history of stroke, focal neurological symptoms and focal neurological signs. The maximum score is 12 , and we excluded any subject with a total score of $>3$. Another exclusion criterion was the presence of silent strokes on the MRI.

Measurement of $\mathrm{BP}$, fasting glucose and $\mathrm{HbA}_{1 \mathrm{c}}$

Sitting BP was measured during one of the visits to our facility. The BP reading was performed at $08.30 \mathrm{~h}$, some $30 \mathrm{~min}$ after the subject arrived. We assessed fasting glucose and $\mathrm{HbA}_{1 \mathrm{c}}$ plasma levels only once during the study; we had no access to longitudinal $\mathrm{HbA}_{1 \mathrm{c}}$ levels. Plasma glucose was measured using a glucose oxidase method (VITROS 950 AT; Amersham, Bucks, UK) and $\mathrm{HbA}_{1 \mathrm{c}}$ using an automated HPLC method (Tosoh Corporation, Kanagawa, Japan) certified by the National Glycohemoglobin Standardization Program.

Magnetic resonance imaging data acquisition and analyses

All cases were blinded for the MRI analyses; raters were unaware of subject identity or group membership. We conducted three types of MRI analyses: white matter pathology was rated; hypotheses-driven volumetric analyses were performed; and, to ensure that we did not miss any potential group differences that we had not hypothesised, a complete brain group comparison was performed using voxel-based morphometry (VBM) analyses (see below for details).

Rating of white matter hyperintensities Fast fluid-attenuated inversion recovery images (TR 9,000 ms, TE $110 \mathrm{~ms}$, TI $2,500 \mathrm{~ms}$, acquisition matrix $154 \times 256$, field of view [FOV] $160 \times 210 \mathrm{~mm}, 20$ slices, slice thickness $5 \mathrm{~mm}$, no gaps, NEX 1, acquisition time $2 \min 15 \mathrm{~s}$ ) were used to rule out primary neurological disease and to quantify white matter disease. We carried out a semiquantitative assessment of white matter hyperintensities (WMHs) using the modified Fazekas scale [33, 34], which assigns scores ranging from 0 to 3 for periventricular hyperintensities and deep WMHs. No fluid-attenuated inversion recovery (FLAIR) scans were available for one subject with diabetes and three control subjects.

Volumetric analysis of the MRI data For the brain measurements, a three-dimensional coronal T1-weighted spoiled gradient recalled sequence (TR $30 \mathrm{~ms}$, TE $2 \mathrm{~ms}, 124$ slices, slice thickness $1.5 \mathrm{~mm}$, no gap, FOV $250 \times 250 \mathrm{~mm}$, matrix $256 \times 128$, flip angle $60^{\circ}$ ) was obtained. This was used to create reformatted images with adequate greywhite matter contrast in order to accurately determine the regional volumes and conduct the voxel-based morphometry analyses (see below).

Regions of interest (ROI) were drawn on reformatted coronal images in the pathological angle using the locally developed Multimodal Image Data Analysis System (MIDAS) software. We outlined individual temporal and frontal lobe structures using our published highly reliable (all inter-rater intra-class correlation coefficients $>0.94$ ) parcellation methods $[35,36]$, briefly outlined below.

1. Hippocampal volume. The hippocampal volume was measured using standardised boundaries [37]. We have validated the hippocampal volumes so derived using post-mortem MRI evaluations [38], and the volumes we obtain are similar to those reported by other investigators [39]. Our image analysis software, MIDAS, allows the simultaneous display of orthogonal views, which makes it easier to identify hippocampus-amygdalar boundaries.

2. Superior temporal gyrus. As a medial border, the superior temporal gyrus has a line joining a reference point in the middle of the temporal horn to the most medial and inferior extension of the Sylvian fissure [35]. The superior margin of this gyrus is the Sylvian fissure. The inferior border is the superior temporal sulcus.

3. Prefrontal region. We used our frontal lobe parcellation method [36] to delineate the prefrontal region. The prefrontal region is bounded anteriorly by the cingulate 
sulcus and posteriorly by the anterior margin of the supplementary motor cortex. By applying a thresholding procedure to the cerebrospinal fluid (CSF) portion of this frontal intracranial volume, we estimated the degree of frontal atrophy.

4. Cerebral vault size. To adjust for individual differences in 'premorbid' brain size and to obtain a measure of overall (global) atrophy, we obtained an intracranial vault volume (ICV) of the supratentorial compartment by following the margins of the dura and tentorium. This estimate of 'premorbid' brain size is used to account for the variability in overall brain size, which may in turn impact on the size of the brain structures of interest. We used a thresholding procedure to estimate the CSF portion of this intracranial vault volume, which was then used as a measure of global atrophy.

5. 'Premorbid' brain size correction. Several methods have been used to adjust for individual differences in 'premorbid' brain size, including the computation of ratios or the use of regression to obtain residualised volumes. A recent review [40] reports that the use of ratios can lead to spurious correlations. Consequently, to adjust for individual differences in 'premorbid' brain size, we residualised all brain volumes to the ICV using regression analyses and then used the residualised volumes in the subsequent statistical analyses. Similarly, the measure of global atrophy, the CSF volume within the intracranial vault, was also residualised to ICV. The prefrontal atrophy measure was obtained by residualising prefrontal CSF to prefrontal intracranial volume. For ease of reference and to allow the comparison of our results to those of other published studies, we also report raw values for all the brain measures in the results section.

Voxel-based morphometry To ensure that our hypothesisdriven manual tracing approach did not miss important group differences in unexpected brain regions, we also ran an automated VBM survey of the brain. VBM analysis was performed with Statistical Parametric Mapping 2 (SPM2) software (Wellcome Department of Imaging Neuroscience, London, UK) on a Windows-based computer. The procedure developed by Good et al. [41] was employed using the default settings.

\section{Statistical analyses}

Two-tailed, independent Student's $t$ tests were used to test for between-group differences in demographic variables and other group descriptors. The $\chi^{2}$ test or Fisher's exact tests were used for nominal variables, as appropriate.

Group differences in cognitive functions were assessed using univariate ANCOVAs with diabetes (yes/no) as a fixed factor and IQ as a covariate. This allowed us to test for cognitive differences independent of overall intelligence.

We tested for group differences in white matter pathology using $\chi^{2}$ tests for the modified Fazekas scale scores. We examined group differences in regional brain volumes using univariate ANOVAs. As described above, residualised brain measures were used for all the statistical analyses involving brain variables.

To investigate the contribution of abnormalities in metabolic parameters typically associated with diabetes ( $\mathrm{HbA}_{1 \mathrm{c}}, \mathrm{BMI}, \mathrm{BP}$ and lipid profiles) on the hypothesised brain differences, we used a multiple regression approach, entering all predictors in a single step. As expected, some subjects in the study were taking medication for the treatment of hypertension or dyslipidaemia. Therefore, we computed dichotomised variables based on the recommendations from the National Cholesterol Education Program (NCEP) guidelines [42]. A subject was thus categorised as hypertensive if he/she received antihypertensive treatment (see Table 1) or had a BP above the NCEP cut-off value (systolic BP $\geq 130 \mathrm{mmHg}$ or diastolic BP $\geq 85 \mathrm{mmHg}$ ). Similarly, a participant was considered dyslipidaemic if he/she was receiving statin treatment or had lipid levels above the NCEP cut-off values (HDL-cholesterol $\leq 1.04 \mathrm{mmol} / 1$ for men, $\leq 1.30 \mathrm{mmol} / 1$ for women, or triacylglycerol $\geq 1.7 \mathrm{mmol} /$ 1). $\mathrm{BMI}, \mathrm{HbA}_{1 \mathrm{c}}$, dyslipidemia (yes/no) and hypertension (yes/no) were then simultaneously entered into a regression model.

The data were analysed using the Statistical Program for Social Sciences (SPSS) version 13.0 (SPSS, Chicago, IL, USA). A $p$ value of less than 0.05 was considered significant. All values are given as means \pm standard deviation (SD).

\section{Results}

Subject groups were well matched in terms of age, sex and education, as illustrated in Table 1. As expected, subjects with diabetes had significantly higher fasting glucose and insulin, $\mathrm{HbA}_{1 \mathrm{c}}$ and $\mathrm{BMI}$, and higher rates of dyslipidaemia and treatment for hypertension. Hamilton depression scores (out of a possible maximum score of 63) were very low (average diabetic patient 3.5 \pm 2.8 ; average control subject $2.3 \pm 2.5$, both of which are well below the clinical cut-off value of 12). Furthermore, Hamilton scores did not correlate with either brain measures or cognition.

\section{Neuropsychological measures}

Although both groups had overall intellectual functioning within the average normal range (Table 1), we found 
Table 1 Descriptive characteristics of individuals with type 2 diabetes and control subjects

\author{
NA Not applicable \\ ${ }^{\mathrm{a}}$ Individuals with diabetes \\ were more likely to be treated \\ for high cholesterol and/or \\ hypertension (HTN)
}

\begin{tabular}{|c|c|c|c|}
\hline & $\begin{array}{l}\text { Type } 2 \text { diabetic subjects } \\
(n=23)\end{array}$ & $\begin{array}{l}\text { Control subjects } \\
(n=23)\end{array}$ & $p$ value \\
\hline Age (years) & $59.2 \pm 8.4$ & $59.9 \pm 8.6$ & 0.68 \\
\hline Education (years) & $15.6 \pm 2.3$ & $15.9 \pm 1.9$ & 0.87 \\
\hline Sex (male/female) & $11 / 12$ & $11 / 12$ & 0.99 \\
\hline Estimated IQ & $107.5 \pm 10.8$ & $114.7 \pm 5.7$ & 0.01 \\
\hline BMI $\left(\mathrm{km} / \mathrm{m}^{2}\right)$ & $31.8 \pm 5.8$ & $24.3 \pm 2.9$ & 0.001 \\
\hline Systolic BP $(\mathrm{mmHg})^{\mathrm{a}}$ & $126.6 \pm 34.3$ & $109.7 \pm 40.5$ & 0.13 \\
\hline Diastolic BP $(\mathrm{mmHg})^{\mathrm{a}}$ & $74.0 \pm 21.5$ & $68.1 \pm 26.2$ & 0.40 \\
\hline HTN treatment (yes/no) & $14 / 9$ & $3 / 20$ & 0.002 \\
\hline Cholesterol $(\mathrm{mmol} / \mathrm{l})^{\mathrm{a}}$ & $4.48 \pm 0.80$ & $4.90 \pm 0.87$ & 0.10 \\
\hline LDL-cholesterol $(\mathrm{mmol} / \mathrm{l})^{\mathrm{a}}$ & $2.59 \pm 0.79$ & $2.97 \pm 0.86$ & 0.13 \\
\hline HDL-cholesterol $(\mathrm{mmol} / \mathrm{l})^{\mathrm{a}}$ & $1.12 \pm 0.27$ & $1.47 \pm 0.36$ & 0.001 \\
\hline Statin treatment (yes/no) & $11 / 12$ & $4 / 19$ & 0.06 \\
\hline Triacylglycerol (mmol/l) & $1.34 \pm 0.36$ & $0.88 \pm 0.09$ & 0.006 \\
\hline $\mathrm{HbA}_{1 \mathrm{c}}(\%)$ & $6.9 \pm 0.8$ & $5.1 \pm 0.4$ & 0.001 \\
\hline Fasting glucose $(\mathrm{mmol} / \mathrm{l})$ & $6.74 \pm 1.84$ & $4.45 \pm 0.45$ & 0.001 \\
\hline Fasting insulin (pmol/1) & $82.65 \pm 51.39$ & $34.73 \pm 8.33$ & 0.001 \\
\hline Time from diagnosis of diabetes (years) & $6.0 \pm 6.3$ & N/A & N/A \\
\hline
\end{tabular}

significant differences in terms of estimated IQ. IQ can affect other cognitive performance, therefore, for completeness, we analysed group differences in the cognitive domains of interest while controlling for IQ. Prior to controlling for IQ, patients with type 2 diabetes had significantly lower scores on all measures of recent (declarative) memory relative to control subjects. The cognitive differences between groups were restricted to recent memory. After IQ adjustment, most of those significant differences remained (Table 2). Groups did not differ (before or after IQ adjustment) on measures of working memory (Digit Span Backward subtest from the WMS-R), executive function (Stroop interference task) or attention (Perceptual Speed, DSST or WMS-R Attention Index).

There were no significant associations between diabetesrelated variables, such as $\mathrm{HbA}_{1 \mathrm{c}}$, and the cognitive variables when the diabetic and control groups were analysed separately.

Brain volumes: between-group analyses

Ratings of WMHs showed infrequent abnormalities overall and no significant group differences for periventricular WMHs (diabetic subjects: score $0, n=3$; score 1: $n=17$, score 2: $n=1$, score $3: n=1$; control subjects: score $0: n=4$, score 1: $n=15$, score $2: n=1$, score $3: n=0 ; \chi^{2}=1.2$, $p=0.76$ ) or deep WMHs (diabetic subjects: score $0: n=9$, score 1: $n=9$, score $2: n=4$, score $3: n=0$; control subjects: score $0: n=9$, score $1: n=7$, score $2: n=4$, score $3: n=0$; $\chi^{2}=0.2, p=0.93$ ).

Voxel-based morphometry (VBM) analysis showed no significant group differences in brain areas outside the hypothesised regions ( $p>0.05$ for all regions, corrected for multiple comparisons). Because of the smoothing used by VBM, we had not anticipated to be able to detect subtle group differences in small brain areas such as the hippocampus with this method. However, using the hypothesis-driven and sensitive manual tracing approach, we detected that patients with type 2 diabetes had significantly smaller hippocampal volumes than the control group (Table 3). No significant differences were observed in global atrophy or volumes of frontal or other temporal regions.

While all correlations of hippocampal volumes and memory tests were positive, none of them reached statistical significance; the Immediate Paragraph score of the Guild Memory Test $(r=0.25, p=0.089)$ and the WMS-R Delayed Index $(r=0.28, p=0.058)$ showed trends.

Associations of hippocampal atrophy and diabetes-related variables

To determine if the observed differences in hippocampal volume were due to non-specific risk factors such as hypertension, dyslipidaemia or obesity, we employed a multiple regression approach.

The regression analysis yielded a significant model ( $R=0.63$, adjusted $\left.R^{2}=0.33, F=6.4, p<0.001\right)$ accounting for $33 \%$ of variance in hippocampal volume. The only significant predictor in this model was $\mathrm{HbA}_{1 \mathrm{c}}$ (standardised $\beta=-0.49, t=-2.9, p<0.01$ ) while BMI (standardised $\beta=$ $-0.18, t=-1.0, p=0.33$ ), hypertension (standardised $\beta=$ $-0.03, t=-0.19, p=0.85$ ), and dyslipidaemia (standardised $\beta=0.01, t=0.05, p=0.96)$ did not significantly contribute to the explained variance. The inverse bivariate association 
Table 2 Cognitive test results of individuals with type 2 diabetes and control subjects

The $p$ values presented are from ANCOVA (controlling for estimated IQ). Note that all recent memory measures were significant prior to controlling for estimated IQ

CVLT California Verbal

Learning Test

${ }^{a}$ Raw scores consisting of number of correct items

${ }^{b}$ Age-corrected scaled scores derived from weighted raw score sums of individual subtests

${ }^{\mathrm{c}}$ Age-corrected raw score

\begin{tabular}{lccc}
\hline & Type 2 diabetic subjects & Control subjects & $p$ value \\
\hline Recent memory and learning & & & \\
WMS-R General Index $^{\mathrm{b}}$ & $117 \pm 12$ & $128 \pm 9$ & 0.05 \\
WMS-R Delayed Index $^{\mathrm{b}}$ & $118 \pm 15$ & $128 \pm 13$ & 0.21 \\
Guild Paragraph Recall-Immediate $^{\mathrm{a}}$ & $5.8 \pm 2.5$ & $7.7 \pm 2.3$ & 0.10 \\
Guild Paragraph Recall-Delayed $^{\mathrm{a}}$ & $7.2 \pm 2.8$ & $9.8 \pm 3.3$ & 0.04 \\
CVLT Short Delay Recall $^{\mathrm{a}}$ & $10.8 \pm 4.1$ & $13.6 \pm 2.0$ & 0.04 \\
CVLT Long Delay Recall $^{\mathrm{a}}$ & $11.4 \pm 3.9$ & $13.9 \pm 1.6$ & 0.02 \\
Working memory $^{\text {Digit Span Backwards }}$ & & & \\
Executive function $^{\mathrm{a}}$ & $8.0 \pm 2.3$ & $7.2 \pm 2.5$ & 0.68 \\
Stroop Interference score $^{\mathrm{c}}$ & & & \\
Attention & $0.07 \pm 8.2$ & $2.8 \pm 9.9$ & 0.63 \\
Digit Symbol Substitution Test & & & \\
Perceptual Speed Correct $^{\mathrm{a}}$ & $52.6 \pm 10.0$ & $53.5 \pm 10.2$ & 0.98 \\
\hline
\end{tabular}

between hippocampal volume and $\mathrm{HbA}_{1 \mathrm{c}}$ is illustrated in Fig. 1. The association between hippocampal volume and $\mathrm{HbA}_{1 \mathrm{c}}$ was also significant within the diabetic group when considered alone ( $r=-0.44, p=0.04$; data not shown).

\section{Discussion}

Our data show that middle-aged individuals with wellcontrolled type 2 diabetes have clear deficits in hippocampal-based (recent or declarative) memory and selective MRI-based atrophy of the hippocampus relative to matched control subjects. Using MRI images of post-mortem brains prior to histological section, we validated our hippocampal volumes by demonstrating that MRI-based hippocampal volumes were equivalent to histological volumes and that the volumes reflected actual neuronal counts [38]. Therefore, the reductions in hippocampal volume we report here among individuals with well-controlled type 2 diabetes may represent neuronal losses in this structure.

We are not aware of any study that has assessed both cognitive functioning and regional brain volumes in individuals with type 2 diabetes. To relate cognition with general brain MRI abnormalities, investigators recently assessed brain atrophy in type 2 diabetic individuals [4, 43]. These studies found attention, executive functions, abstract reasoning and memory impairments to be associated with brain atrophy. However, these studies used semiquantitative estimates of cortical and subcortical atrophy and did not measure regional volumes. Furthermore, these studies evaluated elderly type 2 diabetic subjects with long durations of illness. In contrast, we included individuals who, on average, had been diagnosed with type 2 diabetes for only 6 years and who were in good glycaemic control. In addition, we used a broad imaging approach incorporating automated (VBM), qualitative white matter assessment, as well as volumetric manual tracing procedures. This allowed us to ascertain whether there were group differences in WMHs, grey or white matter density, or global atrophy. Most importantly, our ROI-based volumetric method allowed specific hypothesis testing by use of the sensitive assessment of regional brain volumes of interest such as the hippocampus and prefrontal cortex.

This is the first study to show clear evidence of hippocampal damage (volume losses and impairments in memory and learning) in type 2 diabetes, and may provide a window into very early manifestations of brain complications in type 2 diabetes. The hippocampus is more

Table 3 Brain volumes of individuals with type 2 diabetes and control subjects

\begin{tabular}{lccr}
\hline & Brain volume $\left(\mathrm{cm}^{3}\right)$ & & \\
\cline { 2 - 4 } & Type 2 diabetic subjects & Control subjects & \\
\hline Hippocampus & $5.41 \pm 0.50$ & $6.24 \pm 0.70$ & 0.001 \\
Superior temporal gyrus & $31.37 \pm 5.26$ & $32.27 \pm 4.76$ & 0.659 \\
Frontal lobe & $207.25 \pm 29.62$ & $210.66 \pm 31.85$ & 0.881 \\
Frontal atrophy & $18.49 \pm 8.44$ & $16.91 \pm 6.69$ & 0.394 \\
Global atrophy & $96.87 \pm 35.47$ & $107.36 \pm 90.00$ & 0.713
\end{tabular}

Raw (not residualised) brain volumes (means \pm SD) are given in the table to allow comparability with other studies. Significance values shown are derived from an ANCOVA with residualised brain volumes (see Subjects and methods), having controlled for age 


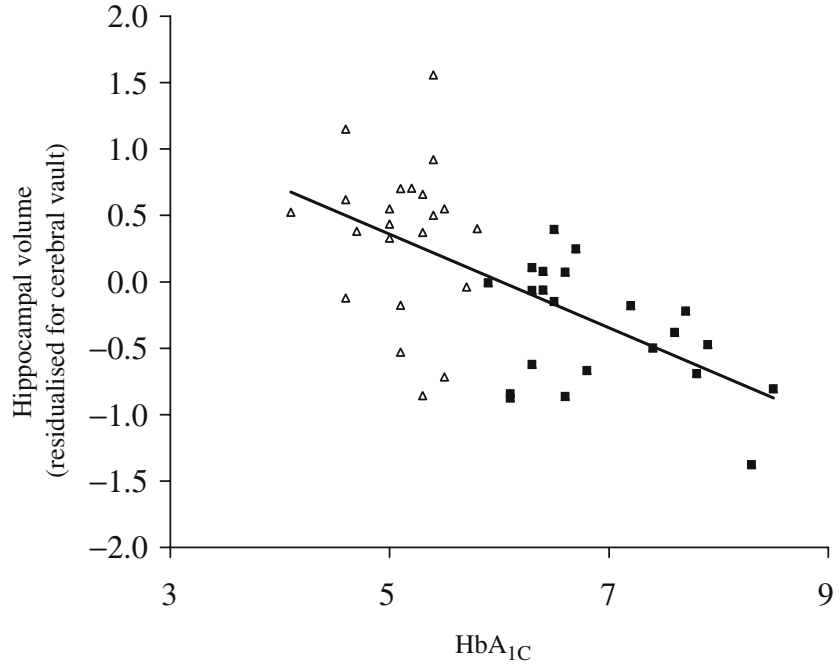

Fig. 1 Bivariate correlations of $\mathrm{HbA}_{1 \mathrm{c}}$ with hippocampal volume (residualised for cerebral vault size). The line shows the line of best fit for the entire study population. Open triangles, control subjects; filled squares, type 2 diabetic subjects. Descriptive characteristics of individuals with type 2 diabetes and control subjects are given in Table 1

susceptible than other brain regions to damage by all sorts of insults, including severe hypoglycaemia and hypoxia $[44,45]$; thus, it is plausible that the hippocampus is the first region to be affected by type 2 diabetes, as our data appear to imply. We propose that, as the disease progresses and other risk factors associated with cardiovascular disease and the metabolic syndrome accumulate, more resilient brain areas are then also affected, as perhaps reflected by the occurrence of global atrophy and white matter pathology. As a result, cognitive impairments may then spread beyond recent memory.

We provide evidence that $\mathrm{HbA}_{1 \mathrm{c}}$, which reflects how well diabetes has been controlled over the previous 2-3 months, is specifically associated with the hippocampal volume losses in the population studied. Other variables commonly associated with type 2 diabetes, such as elevated BMI, hypertension or dyslipidaemia, did not offer an independent contribution to explaining variance in hippocampal volume; their contributions were subsumed by $\mathrm{HbA}_{1 \mathrm{c}}$. Furthermore, our results are not confounded by gross white matter abnormalities, increased global atrophy or age.

These findings imply that the insulin resistance and accompanying poor glucose control, which are at the core of type 2 diabetes, may be responsible for the associated early hippocampal volume reductions. Moreover, these findings argue for the specificity of the associations between type 2 diabetes and the brain and cognitive abnormalities seen, beyond the possible non-specific contributions of the various indices of the metabolic syndrome.

It is becoming clear that diabetes, whether it is type 1 or type 2 , has a negative impact on both cognition and brain.
However, the resultant adverse effects may vary according to the type of diabetes. Whereas most studies on type 1 diabetes report deficits in processing speed, intelligence and attention, those on type 2 diabetes mostly show impairments in verbal memory and processing speed, with relative preservation of visual and spatial function and attention. Even a study that evaluated MRI volumes and cognition in the same set of relatively young type 1 diabetic subjects (30-50 years of age with disease durations of $>20$ years) [46], failed to demonstrate differences in memory or hippocampus volume relative to control subjects. Instead, they found increased cerebral atrophy and deficits in attention and psychomotor speed. While both type 1 and type 2 diabetes share hyperglycaemia as a manifestation of the illness, type 2 diabetes is characterised by insulin resistance even during the preclinical stages (prediabetes). Perhaps it is this key difference between type 1 and type 2 diabetes that contributes to the differential brain consequences of these two disorders. This position is, in part, supported by our prior data showing associations between insulin resistance and lower memory performance and hippocampal volumes among non-diabetic individuals [22].

To date, little is known about the mechanisms that contribute to regionally specific brain tissue loss in type 2 diabetes. Some investigators have proposed that the tissue damage in diabetes may be the consequence of increased oxidative stress resulting from increased availability of metabolic substrate [47]. We have postulated an alternative mechanism based on the endothelial dysfunction known to accompany insulin resistance (and type 2 diabetes). Investigators have demonstrated that there are specific interstitial decreases in glucose levels in those brain areas activated [48]. Given that the glucose transporter at the blood-brain barrier is saturated at physiological glucose levels, to bring more glucose to the activated region acutely, new glucose transporters need to be exposed to the blood. Therefore, intact endothelial function is likely needed to recruit new capillaries to carry additional glucose to the activated sites, and its dysfunction could result in functional hypoglycaemia during periods of increased demand. This relative functional hypoglycaemia, when coupled with the elevations in cortisol levels that are often present in diabetes, may result in tissue damage, particularly in those brain regions more vulnerable to damage, such as the hippocampus [45].

Some limitations of this study should be taken into consideration when interpreting our results. First, our sample was relatively small, which affects the generalisability as well as the power of the study. However, the sample size was sufficient to detect significant differences in memory, as well as hippocampal volumes, illustrating the strength of these associations with type 2 diabetes. Second, the sample was heterogeneous because of the inclusion of 
participants who were receiving BP or lipid-lowering therapies. We accounted for this potentially masking effect of treatment by using dichotomous variables for hypertension and dyslipidaemia. Since high BP and dyslipidaemia are common in type 2 diabetes, we believe that our approach actually increases the generalisability of our findings. However, in our sample we were not able to take into account the degree of success of these pharmacological interventions. This should be addressed in future studies. Last, our sample only showed weak positive associations between hippocampal volumes and memory test performance. This is in line with a recent meta-analysis [40], which found little evidence for a strong direct correlation.

Future longitudinal studies should focus on enrolling subjects before they meet diagnostic criteria for type 2 diabetes (in the prediabetic stage), and follow them during their transition into diabetes, which may provide further insight into the pathological brain mechanisms at work. Furthermore, future research should include more comprehensive longitudinal evaluation of endocrine status (glucose regulation as well as hypothalamic-pituitary-adrenal axis), as well as other possible aetiological or modifying variables, both damaging (e.g. hypertension, lipid profiles and pro-inflammatory markers) and protective (e.g. brainderived neurotrophic factor). Furthermore, future human studies could directly assess brain energetics through the use of MRI-based spectroscopy or glucose positron emission tomography. This comprehensive approach will add to our understanding of the mechanisms that may contribute to the damage present in insulin resistance and type 2 diabetes.

Acknowledgements The study was supported by grants from the National Institutes of Health (DK064087 and P30-AG-08051) and support from the New York University General Clinical Research Center (NCRR M01 RR00096). S. Gold was, in part, supported by a post-doctoral fellowship from the German Research Society (GO-1357/1-1).

Duality of interest None of the authors have any financial interest in this work.

\section{References}

1. Cowie CC, Rust KF, Byrd-Holt DD et al (2006) Prevalence of diabetes and impaired fasting glucose in adults in the U.S. population: National Health and Nutrition Examination Survey 1999-2002. Diabetes Care 29:1263-1268

2. Strachan M, Deary I, Ewing F, Frier B (2002) Is type II diabetes associated with an increased risk of cognitive dysfunction? A critical review of published studies. Diabetes Care 20:438-445

3. Biessels G-J, ter Braak E, Erkelens D, Hijman R (2001) Cognitive function in patients with type 2 diabetes mellitus. Neurosci Res Commun 28:11-22

4. Manschot SM, Brands AMA, van der Grond J et al (2006) Brain magnetic resonance imaging correlates of impaired cognition in patients with type 2 diabetes. Diabetes 55:1106-1113
5. Ryan CM, Geckle MO (2000) Circumscribed cognitive dysfunction in middle-aged adults with type 2 diabetes. Diabetes Care 23:1486-1493

6. Araki Y, Nomura M, Tanaka H et al (1994) MRI of the brain in diabetes mellitus. Neuroradiology 36:101-103

7. den Heijer T, Vermeer SE, van Dijk EJ et al (2003) Type 2 diabetes and atrophy of the medial temporal lobe structures. Diabetologia 46:1604-1610

8. Korf ESC, White LR, Scheltens P, Launer LJ (2006) Brain aging in very old men with type 2 diabetes: the Honolulu-Asia aging study. Diabetes Care 29:2268-2274

9. Kumari M, Brunner E, Fuhrer R (2000) Minireview: mechanisms by which the metabolic syndrome and diabetes impair memory. J Gerontol 55A:B228-B232

10. Reaven GM, Thompson LW, Nahum D, Haskins E (1990) Relationship between hyperglycemia and cognitive function in older NIDDM patients. Diabetes Care 13:16-21

11. Schneider D, Sobel B (1997) Determinants of coronary vascular disease in patients with type II diabetes mellitus and their therapeutic implications. Clin Cardiol 20:433-440

12. Garg A (1998) Treatment of diabetic dyslipidemia. Am J Cardiol 81:47B-51B

13. Taskinen M, Smith U (1998) Lipid disorders in NIDDM: implications for treatment. J Intern Med 244:361-370

14. Jellinger P (2000) The American Association of Clinical Endocrinologists medical guidelines for clinical practice for the diagnosis and treatment of dyslipidemia and prevention of atherogenesis. Endocr Pract 6:162-213

15. Cournot M, Marquie JC, Ansiau D et al (2006) Relation between body mass index and cognitive function in healthy middle-aged men and women. Neurology 67:1208-1214

16. Ward MA, Carlsson CM, Trivedi MA, Sager MA, Johnson SC (2005) The effect of body mass index on global brain volume in middle-aged adults: a cross sectional study. BMC Neurol 5:23

17. Gold SM, Dziobek I, Rogers K, Bayoumy A, McHugh PF, Convit A (2005) Hypertension and hypothalamo-pituitary-adrenal axis hyperactivity affect frontal lobe integrity. J Clin Endocrinol Metab 90:3262-3267

18. Papademetriou V (2005) Hypertension and cognitive function. Blood pressure regulation and cognitive function: a review of the literature. Geriatrics 60:20-22, 24

19. Meneilly GS, Cheung E, Tessier D, Yakura C, Tuokko H (1993) The effect of improved glycemic control on cognitive functions in the elderly patient with diabetes. J Gerontol 48:M117-M121

20. Gradman TJ, Laws A, Thompson LW, Reaven GM (1993) Verbal learning and/or memory improves with glycemic control in older subjects with non-insulin-dependent diabetes mellitus. J Am Geriatr Soc 41:1305-1312

21. Vanhanen M, Koivisto K, Karjalainen L et al (1997) Risk for noninsulin-dependent diabetes in the normoglycaemic elderly is associated with impaired cognitive function. NeuroReport 8:1527-1530

22. Convit A, Wolf OT, Tarshish C, de Leon MJ (2003) Reduced glucose tolerance is associated with poor memory performance and hippocampal atrophy among normal elderly. Proc Natl Acad Sci U.S.A. 100:2019-2022

23. Kalmijn S, Feskens EJM, Launer LJ, Stijnen T, Kromhout D (1995) Glucose intolerance, hyperinsulinaemia and cognitive function in a general population of elderly men. Diabetologia 38:1096-1102

24. Delis DC, Kramer JH, Kaplan E, Ober BA (1987) California verbal learning test-research edition. The Psychological Corporation, New York

25. Wechsler D (1987) Wechsler memory scale-revised. Psychological Corporation/Harcourt Brace Javanovich, San Antonio

26. Gilbert JG, Levee RF, Catalano FL (1968) A preliminary report on a new memory scale. Percept Mot Skills 27:277-278 
27. Golden CJ (1978) Stroop color and word test: a manual for clinical and experimental uses. Stoeiting Company, Wood Dale

28. Zachary RA (1940) Shipley Institute of living scale-revised. Western Psychological Services, Los Angeles

29. Zachary RA, Paulson MJ, Gorsuch RL (1985) Estimating WAIS IQ from the Shipley Institute of Living Scale using continuously adjusted age norms. J Clin Psychol 41:820-831

30. Marcus S, Robins LN, Bucholz K (1988) Quick diagnostic interview schedule III-R. Version 1.0. Washington University School of Medicine, St Louis

31. Hamilton M (1960) A rating scale for depression. J Neurol Neurosurg Psychiatry 23:56-62

32. Rosen WG, Terry RD, Fuld PA, Katzman R, Peck A (1980) Pathological verification of ischemia score in differentiation of dementias. Ann Neurol 7:486-488

33. Fazekas F, Chawluk JB, Alavi A, Hurtig HI, Zimmerman RA (1987) MR signal abnormalities at $1.5 \mathrm{~T}$ in Alzheimer's dementia and normal aging. Am J Roentgenol 149:351-356

34. Scheltens P, Barkhof F, Leys D et al (1993) A semiquantative rating scale for the assessment of signal hyperintensities on magnetic resonance imaging. J Neurol Sci 114:7-12

35. Convit A, de Leon MJ, Tarshish C et al (1997) Specific hippocampal volume reductions in individuals at risk for Alzheimer's disease. Neurobiol Aging 18:131-138

36. Convit A, Wolf OT, de Leon MJ et al (2001) Volumetric analysis of the pre-frontal regions: findings in aging and schizophrenia. Psychiatry Res 107:61-73

37. Convit A, McHugh PR, Wolf OT et al (1999) MRI volume of the amygdala: a reliable method allowing separation from the hippocampal formation. Psychiatry Res 90:113-123

38. Bobinski M, de Leon MJ, Wegiel J et al (2000) The histological validation of post mortem magnetic resonance imaging-determined hippocampal volume in Alzheimer's disease. Neurosci 95:721-725
39. Jack CRJ, Bentley MD, Twomey CK, Zinsmeister AR (1990) MR imaging-based volume measurements of the hippocampal formation and anterior temporal lobe: validation studies. Radiology 176:205-209

40. Van Petten C (2004) Relationship between hippocampal volume and memory ability in healthy individuals across the lifespan: review and meta-analysis. Neuropsychologia 42:1394-1413

41. Good CD, Johnsrude IS, Ashburner J, Henson RNA, Friston KJ, Frackowiak RSJ (2001) A voxel-based morphometric study of ageing in 465 normal adult human brains. NeuroImage 14:21-36

42. Expert Panel (2001) Executive Summary of the Third Report of the National Cholesterol Education Program (NCEP) Expert Panel on Detection, Evaluation and Treatment of High Blood Cholesterol in Adults (Adult Treatment Panel III). JAMA 285:2486-2497

43. Akisaki T, Sakurai T, Takata T et al (2006) Cognitive dysfunction associates with white matter hyperintensities and subcortical atrophy on magnetic resonance imaging of the elderly diabetes mellitus Japanese elderly diabetes intervention trial (J-EDIT). Diabetes Metab Res Rev 22:376-384

44. Burke SN, Barnes CA (2006) Neural plasticity in the ageing brain. Nat Rev Neurosci 7:30-40

45. Convit A (2005) Links between cognitive impairment in insulin resistance: an explanatory model. Neurobiol Aging 26:31-35

46. Lobnig BM, Kroemeke O, Optenhostert-Porst C, Wolf OT (2006) Hippocampal volume and cognitive performance in long-standing type 1 diabetic patients without macrovascular complications. Diabet Med 23:32-39

47. Brownlee M (2001) Biochemistry and molecular cell biology of diabetic complications. Nature 414:813-820

48. McNay EC, Fries TM, Gold PE (2000) Decreases in rat extracellular hippocampal glucose concentration associated with cognitive demand during a spatial task. Proc Natl Acad Sci 97:2881-2885 